\title{
Spread of a highly mucoid Streptococcus pyogenes emm3/ST15 clone
}

\author{
Esther Tamayo ${ }^{1}$, Milagrosa Montes ${ }^{1,2}$, Guadalupe García-Medina², José M García-Arenzana², \\ Emilio Pérez-Trallero $1,2,3^{*}$
}

\begin{abstract}
Background: Hyaluronic acid capsule plays a key role in Streptococcus pyogenes virulence. Circulation of mucoid or highly encapsulated strains has been related to rheumatic fever epidemics and invasive disease in several countries. In 2009, an outbreak of mucoid S. pyogenes isolates was detected in northern Spain. The aim of the study was to describe clinical and molecular characteristics of mucoid strains causing this outbreak and to compare them with a sample of non-mucoid S. pyogenes isolates obtained during the same period of time.
\end{abstract}

Methods: All S. pyogenes isolates with a mucoid colony morphology $(n=132), 10 \%$ of non-mucoid $(n=144)$ and all invasive S. pyogenes isolates $(n=7)$ obtained in 2009 were included. Characterization was performed by T-agglutination, emm typing, pulsed field gel electrophoresis and multilocus sequence typing.

Results: One clone characterized as emm3.1/ST15 comprised $98.5 \%(n=130)$ of all mucoid isolates. Subjects of all ages were affected. Main clinical manifestations were pharyngitis and scarlet fever, but this clone also caused invasive disease: two cases of streptococcal toxic shock syndrome, one arthritis, and one celullitis with a fatal outcome. Mucoid isolates were more prone to cause invasive disease than non-mucoid isolates ( $p=0.001)$.

Conclusions: Although no acute rheumatic fever cases were detected, the most worrisome characteristics of this clone were the success for causing invasive disease and the merge of two virulent features: the serotype, emm3, and capsule hyper-production, expressed as a mucoid morphology.

\section{Background}

Group A streptococcus (GAS) strains differ widely in their encapsulation degree, and those with an exuberant capsule production have a mucoid morphology when are cultured on blood agar plates. The expression of the capsule has been related to the virulence of the strain since it was first recognized [1,2]. In the United States temporal association between a high incidence of rheumatic fever cases and an increased presence of mucoid S. pyogenes strains was observed [3]. More concretely, the rheumatogenic ability has been associated to a limited number of emm types such as emm1,3,5, 6, or 18 which often were mucoid $[1,3]$ although this pattern is not held in all parts of the world, like in Hawaii [4]. Furthermore, these emm types, and particularly emm 1 and emm 3 types, are the most prevalent $\mathrm{emm}$ types found among

\footnotetext{
* Correspondence: mikrobiol@terra.es
'Biomedical Research Centre Network for Respiratory Diseases (CIBERES), San

* Correspondence: mikrobiol@terra.es
'Biomedical Research Centre Network for Respiratory Diseases (CIBERES), San Sebastián, Spain
}

() 2010 Tamayo et al; licensee BioMed Central Ltd. This is an Open Access article distributed under the terms of the Creative Commons Attribution License (http://creativecommons.org/licenses/by/2.0), which permits unrestricted use, distribution, and reproduction in any medium, provided the original work is properly cited.
By performing blood agar cultures for $S$. pyogenes detection, phenotypic characteristics like mucoid colony morphology can be recognized. In fact, recently the advisability of detecting mucoid isolates in clinical practice has been emphasized in the literature [1].

In 2009, a sudden emergence of encapsulated S. pyogenes isolates was observed among clinical samples received at the microbiology laboratory of Hospital Donostia, (Gipuzkoa), in Northern Spain, where circulation of mucoid S. pyogenes strains was highly infrequent in the past. The aim of this work was to describe molecular characteristics of mucoid isolates causing this outbreak and to compare them to a sample of non-mucoid $S$. pyogenes isolates obtained in the same period of time.

\section{Methods}

\section{Geographical location}

The province of Gipuzkoa is situated in the north of Spain, flanked by France in the north. Hospital 
Donostia, in Gipuzkoa, is the referral hospital for the province, and attends a population of 405,745 inhabitants of all ages, children included. The microbiology laboratory receives all throat samples from Primary Care Centers of the area to investigate the presence of GAS.

\section{Isolate sample and identification}

All S. pyogenes isolates with a mucoid colony morpho$\operatorname{logy}(n=132)$ detected in 2009 were included in the study. Also, randomly selected $10 \%$ of non-mucoid S. pyogenes isolates from pharyngeal swabs $(\mathrm{n}=144)$ and all S. pyogenes isolates from sterile body sites $(\mathrm{n}=7)$ obtained that year were analyzed. To investigate the presence of GAS, samples were routinely cultured on blood agar plates and incubated overnight in an atmosphere enriched with $5 \%$ of $\mathrm{CO}_{2}$ at $37^{\circ} \mathrm{C}$. Colonies were identified as GAS by bacitracin-susceptibility and agglutination with specific Group-A streptococci antisera (Slidex Strepto-kit; bioMérieux, Marcy l'Etoile, France).

\section{Isolate characterization}

Characterization was performed by $\mathrm{T}$-agglutination, emm typing, pulsed field gel electrophoresis (PFGE) and multilocus sequence typing (MLST) as described elsewhere [7]. T-type was done by agglutination of trypsin digested bacteria suspensions using commercial poly- and mono-specific $\mathrm{T}$ antisera according to manufacturer's instructions (Sevapharma, Prague, Czech Republic). emm-type was determined by polymerase chain reaction-restriction fragment length polimorfism (PCR-RFLP) assay. In those isolates were emm gene did not amplify, specific recommendations with a lysate preparation for mucoid strains were followed (available at: http://www.cdc.gov/ncidod/biotech/strep/protocol_emm-type.htm). The emm-gene of all invasive isolates, and at least $15 \%$ of each different emm-type of non-invasive (mucoid and non-mucoid) isolates were sequenced according to the guidelines of the Division of Bacterial and Mycotic Diseases, Centers for Disease Control and Prevention (CDC) (available at: http://www.cdc.gov/ncidod/biotech/strep/MProteinGene_typing.htm). All mucoid isolates ( $\mathrm{n}=$ 132) and all non-mucoid emm3 $(\mathrm{n}=24)$ isolates were investigated by PFGE. Samples were digested with SmaI enzyme, and results were analyzed by the Diversity Database fingerprinting software, version 2 (BioRad, USA) to construct a dendrogram by the unweighted pair group method with arithmetic averages, Dice coefficient, and a position tolerance of $1 \%$. Isolates belonging to the same PFGE pattern had a similarity higher than $85 \%$. A sample representing at least $10 \%$ of each PFGE pattern ( $\mathrm{n}=18$ isolates) was characterized by MLST (available at: http://spyogenes. mlst.net/misc/info.asp).

\section{Antibiotic susceptibility testing}

Erythromycin, clindamycin, tetracycline and levofloxacin susceptibility testing was performed by broth microdilution method, using Sensititre microtitre trays (Trek Diagnostic Systems, East Sussex, UK) with Mueller-Hinton II broth (BioMerieux, Mercy l'Etoile, France) supplemented with lysed horse blood (3-5\% vol/vol). Minimum inhibitory concentrations (MICs) were performed and interpreted according to the criteria recommended by the Clinical and Laboratory Standards Institute (CLSI) [8].

\section{Statistical analysis}

Data were analyzed with the Instat3 program. Chi square and Fisher's exact probability tests were used to perform comparisons.

\section{Ethics Statement}

In the present study no human experimentation was conducted, with all studies carried out on microorganisms. The study and publication of their results was approved by the 'Comité Ético de Investigación Clínica del Área Sanitaria de Gipuzkoa'.

\section{Results and Discussion}

In 2009, 1571 S. pyogenes isolates were detected among samples received at the microbiology laboratory of Hospital Donostia. Of them, 132 were mucoid, representing the $8.4 \%$ of all isolates of that year, while in the previous four years only 9 mucoid isolates were detected. Circulation of mucoid isolates through 2009 is represented in figure 1.

Crater et al. [9] demonstrated that capsule production is greatest in the early logarithmic growth phase in broth cultures and that its expression is lost when the stationary growth phase is reached. In our experience, mucoid colonies showed a wet and glossy shape in blood agar plates (figure 2), although few of them were already dried showing a colapsed, rough and "matte" morphology different to that found in non-mucoid colonies. Incubation of blood agar plates in an atmosphere enriched with $5 \%$ of $\mathrm{CO}_{2}$ enhanced capsule expression. All mucoid isolates were susceptible to erythromycin, clindamycin, tetracycline and levofloxacin antimicrobials showing the following MICs: erythromycin MIC $<0.5$ $\mu \mathrm{g} / \mathrm{mL}$; clindamycin $\mathrm{MIC}<0.5 \mu \mathrm{g} / \mathrm{mL}$; tetracycline MIC $<4 \mu \mathrm{g} / \mathrm{mL}$; and levofloxacin MIC $<2 \mu \mathrm{g} / \mathrm{mL}$.

Overall, $98.5 \%$ of all mucoid isolates (130/132) belonged to the emm3.1 type. The two isolates with a different emm type were characterized as emm5.46 and emm1.0. In a high percentage of mucoid isolates the emm gene was difficult to amplify. Probably the vast amount of hyaluronic acid could have interfered in DNA extraction as after following specific CDC 


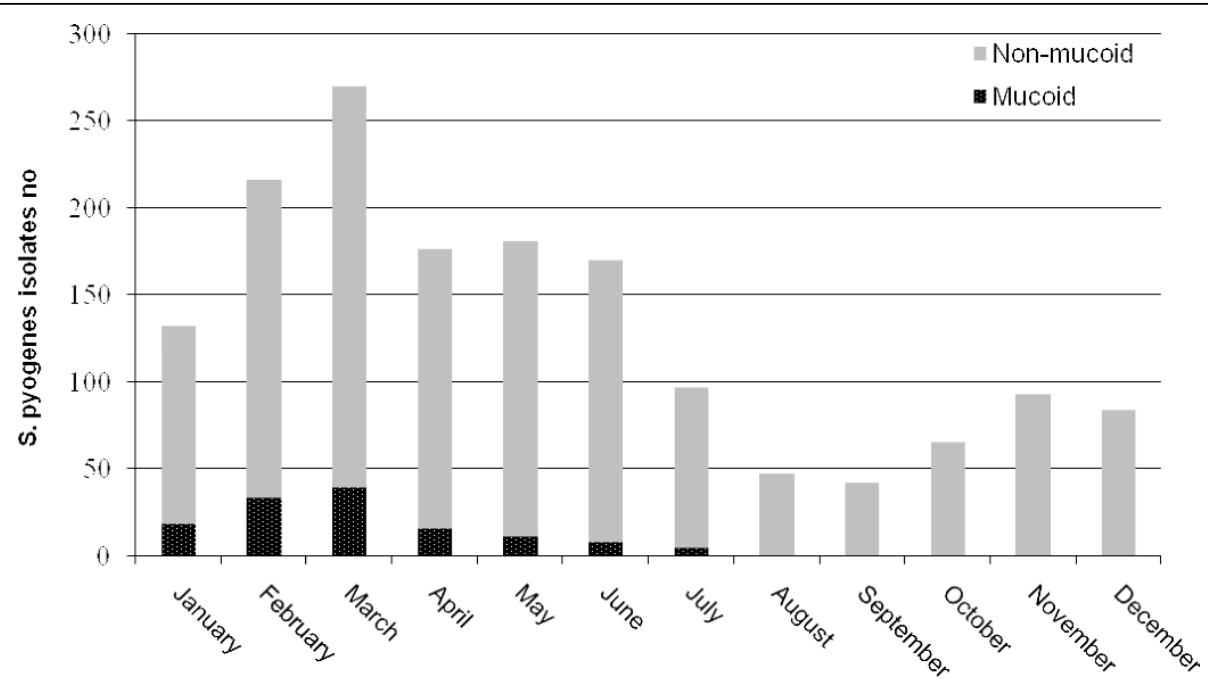

Figure 1 Circulation of mucoid and non-mucoid S. pyogenes isolates in $2009(n=1571)$.

recommendations for mucoid strains, a complete $\mathrm{emm}$ gene amplification success was obtained. PFGE revealed 4 different patterns: patterns $\mathrm{A}$ and $\mathrm{B}$, with a similarity of $73 \%$ between them for emm 3 mucoid isolates (figure 3), pattern $\mathrm{C}$ for the emm 1 isolate and pattern $\mathrm{D}$ for the emm5 isolate. All emm3 mucoid isolates, independently of the PFGE pattern, belonged to the ST15, the emm1 isolate to the ST28 and the emm5 isolate to the ST99. All emm3 isolates were non T-typeable. This finding was unrelated to the mucoid capsule because among 89 non-mucoid emm 3 isolates obtained in the last 4 years, only 1 could be T-typed as T3 (data not shown). The circulation of a mucoid S. pyogenes clone (emm3.1/ ST15) in 2009 contrasts with the results obtained in the past, when only a few mucoid isolates representing a

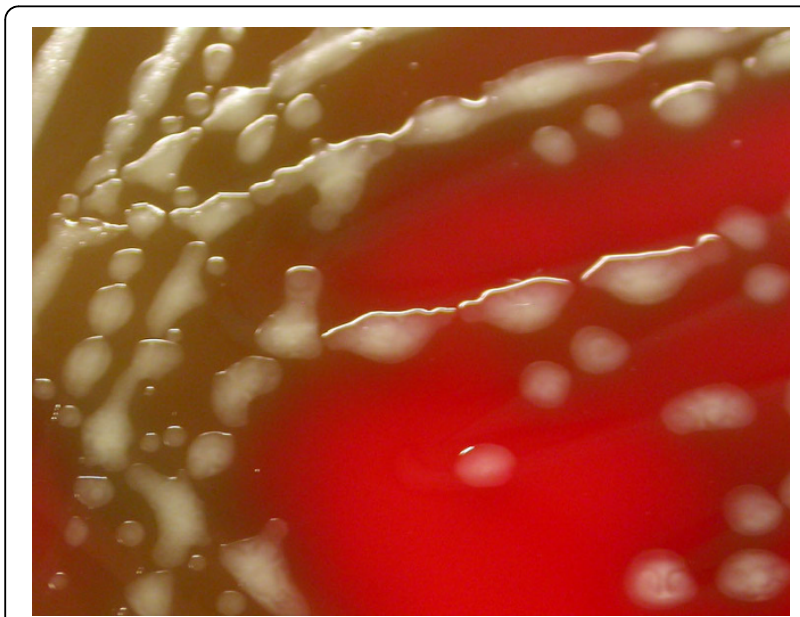

Figure 2 Mucoid S. pyogenes colonies on blood agar plates after overnight incubation under an atmosphere enriched with $5 \% \mathrm{CO}_{2}$. varied sample of $\mathrm{emm}$ and sequence types were detected in the community.

Twenty four out of 144 non-mucoid isolates analyzed in 2009, belonged to the emm3 type, which shared the same molecular and susceptibility characteristics (emm3.1/ST15) as the emm3 mucoid isolates, although belonged only to the PFGE pattern A. The remaining 120 non-emm3 non-mucoid isolates showed percentages of non-susceptibility in variable proportions $(19.2 \%$ to erythromycin, $5.8 \%$ to clindamycin, $5.8 \%$ to tetracycline and $1.7 \%$ to levofloxacin). The only difference between emm3 non-mucoid and mucoid isolates was the degree of hyaluronate encapsulation.

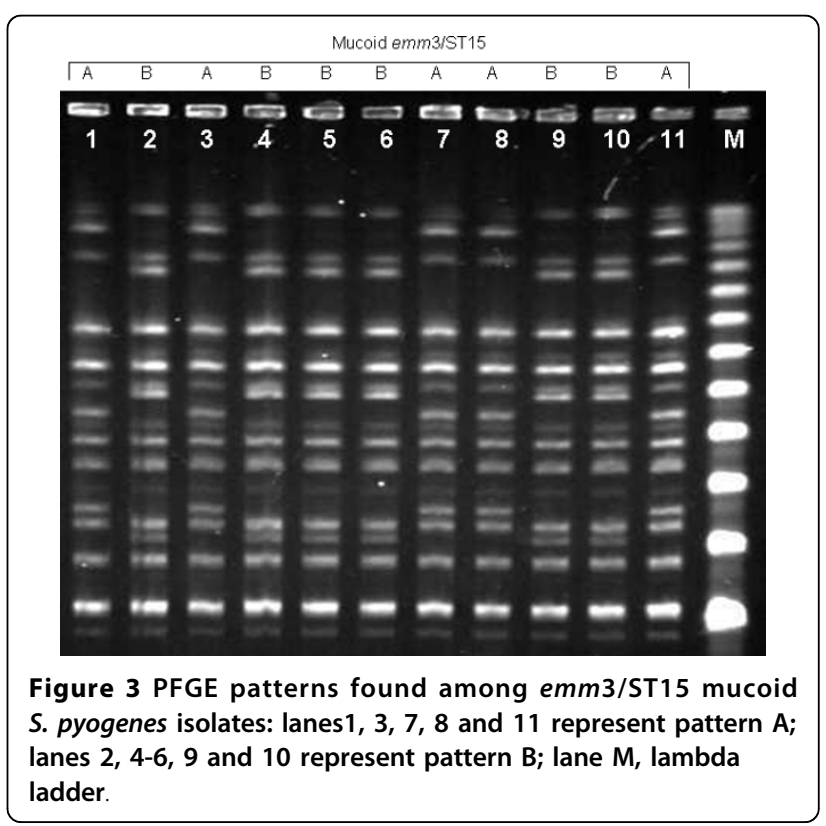


Among mucoid isolates, $87.9 \%(\mathrm{n}=116)$ were obtained from throat samples. Age distribution of patients affected by the mucoid clone was similar to that among patients infected by non-mucoid $S$. pyogenes isolates: $84.1 \%(\mathrm{n}=111)$ and $80.2 \%(\mathrm{n}=1154)$ were children under 14 years old respectively $(\mathrm{p}=0.30)$. The main clinical manifestations were pharyngitis $(\mathrm{n}=55)$, scarlet fever $(\mathrm{n}=30)$ and otitis $(\mathrm{n}=13)$, similar to those found among non-mucoid isolates $(\mathrm{n}=1439)$, although they also caused 4 cases of invasive disease. The emm 3 type has been ranked among the most prevalent emm-types isolated from patients with severe disease worldwide and has been related to high case fatality rates [5,6]. In this study four emm 3 mucoid isolates were obtained from sterile body sites (3 from blood and 1 from pleural fluid), two of them associated with streptococcal toxic shock syndrome, 1 with arthritis, and 1 with celullitis with a fatal outcome. Among non-mucoid isolates $(n=1439)$, only 3 cases of invasive disease were registered (one emm3.1, one emm75, and one emm87). These data revealed that invasive disease was more often caused due to mucoid isolates (3\%) compared to non-mucoid isolates $(0.21 \%, \mathrm{p}=0.001)$ in 2009 in our hospital. Independently of the mucoid feature, the emm 3 type was detected in $71.4 \%$ of all invasive GAS disease of that year.

We have found few reports investigating mucoid $S$. pyogenes colony phenotype in Spain. Bosch et al. [10] reported 11 out of 31 invasive $S$. pyogenes isolates obtained between 1995 and 1998 showing a mucoid appearance, although only three were heavily mucoid. Sierra et al. [11] investigated the presence of mucoid isolates among invasive GAS infections in injecting drug users in Barcelona, finding that all isolates were non-mucoid. By contrast, in other parts of the world, like in the United States, mucoid GAS circulation has been more frequently reported. Veasy et al. [3] found an association between mucoid strains circulation and an increase of acute rheumathic fever incidence. Rheumatic fever is considered a rare disease in Europe [12] and no cases were produced in our region in the last ten years. Nevertheless, after the characterization of this clone which merges a traditionally virulent serotype (emm3) and the mucoid feature, pediatricians and other physicians were alerted in order to prevent the re-emergence of rheumatic fever into an area previously free of this disease. We have investigated the epidemiology of $S$. pyogenes in our community since the 90 's, and to our knowledge emm3 S. pyogenes isolates circulate every year but without showing a mucoid appearance (data not shown). Why these emm3 S. pyogenes isolates express the mucoid phenotype is under research.

\section{Conclusions}

To our knowledge, this is the first outbreak of mucoid S. pyogenes isolates described in the community in southern Europe, and reminds us of the importance of performing a comprehensive phenotypic and genotypic characterization of circulating strains. It is of special concern that the same isolate merged two important virulence factors: the emm-type, emm3, and the hiperproduction of capsule observed as a mucoid morphology, which could have enhanced the ability to cause severe disease.

\section{Acknowledgements}

This work was supported by the grants PI 080808 from the Ministerio de Ciencia e Innovación, Spain and GIU09-59 from the University of the Basque Country, UPV/EHU, Spain.

\section{Author details}

${ }^{1}$ Biomedical Research Centre Network for Respiratory Diseases (CIBERES), San Sebastián, Spain. ${ }^{2}$ Microbiology Service, Hospital Donostia, San Sebastián, Spain. ${ }^{3}$ Department of Preventive Medicine and Public Health, Faculty of Medicine, Basque Country University, San Sebastián, Spain.

\section{Authors' contributions}

EPT designed and coordinated the experiments. ET, MM, GGM, JMGA performed the experiments, sequence analysis and analyzed the data. EPT, MM and ET drafted the manuscript. All authors read and approved the final manuscript.

\section{Competing interests}

The authors declare that they have no competing interests.

Received: 31 May 2010 Accepted: 5 August 2010

Published: 5 August 2010

\section{References}

1. Stollerman $\mathrm{GH}$, Dale JB: The importance of the group a streptococcus capsule in the pathogenesis of human infections: a historical perspective. Clin Infect Dis 2008, 46:1038-1045.

2. Wessels MR, Moses AE, Goldberg JB, DiCesare TJ: Hyaluronic acid capsule is a virulence factor for mucoid group A streptococci. Proc Natl Acad SCi USA 1991, 88:8317-8321.

3. Veasy LG, Tani LY, Daly JA, Korgenski K, Miner L, Bale J, Kaplan EL, Musser JM, Hill HR: Temporal association of the appearance of mucoid strains of Streptococcus pyogenes with a continuing high incidence of rheumatic fever in Utah. Pediatrics 2004, 113:e168-e172.

4. Erdem G, Mizumoto C, Esaki D, Reddy V, Kurahara D, Yamaga K, Abe L, Johnson D, Yamamoto K, Kaplan EL: Group A streptococcal isolates temporally associated with acute rheumatic fever in Hawaii: differences from the continental United States. Clin Infect Dis 2007, 45:e20-e24.

5. Luca-Harari B, Darenberg J, Neal S, Siljander T, Strakova L, Tanna A, Creti R, Ekelund K, Koliou M, Tassios PT, van der Linden M, Straut M, VuopioVarkila J, Bouvet A, Efstratiou A, Schalén C, Henriques-Normark B, StrepEURO Study Group, Jasir A: Clinical and microbiological characteristics of severe Streptococcus pyogenes disease in Europe. J Clin Microbiol 2009, 47:1155-1165.

6. O'Loughlin RE, Roberson A, Cieslak PR, Lynfield R, Gershman K, Craig A, Albanese BA, Farley MM, Barrett NL, Spina NL, Beall B, Harrison LH, Reingold A, Van Beneden C, Active Bacterial Core Surveillance Team: The epidemiology of invasive group A streptococcal infection and potential vaccine implications: United States, 2000-2004. Clin Infect Dis 2007, 45:853-862.

7. Pérez-Trallero E, Montes M, Orden B, Tamayo E, García-Arenzana JM, Marimón JM: Phenotypic and genotypic characterization of Streptococcus pyogenes isolates displaying the MLSB phenotype of 
macrolide resistance in Spain, 1999 to 2005. Antimicrob Agents Chemother 2007, 51:1228-1233.

8. Clinical and Laboratory Standards Institute: Performance standards for antimicrobial susceptibility testing; 19th informational suplement. CLSI documents M100-S19 2009.

9. Crater DL, van de Rijn I: Hyaluronic acid synthesis operon (has) expression in group A streptococci. J Biol Chem 1995, 270:18452-18458.

10. Bosch T, Riera M, Galmés M, Ramírez A, Albertí S: [Invassive infections caused by Group A streptococci: clinical characteristics and microbiological analyses in 31 cases]. Enferm Infecc Microbiol Clin 2001, 19:371-375.

11. Sierra JM, Sánchez F, Castro P, Salvadó M, de la Red G, Libois A, Almela M, March F, Español M, Sambeat MA, Romeu J, Brugal MT, García de Olalla P, Gatell JM, Vila J, García F, López Colomés JL, Caylá JA, Coll P: Group A streptococcal infections in injection drug users in Barcelona, Spain: epidemiologic, clinical, and microbiologic analysis of 3 clusters of cases from 2000 to 2003. Medicine (Baltimore) 2006, 85:139-146.

12. Weil-Olivier C: Rheumatic fever. Orphanet encyclopedia 2004 [http://www. orpha.net/data/patho/GB/Uk-RF.pdf], (read May 2010).

\section{Pre-publication history}

The pre-publication history for this paper can be accessed here: http://www.biomedcentral.com/1471-2334/10/233/prepub

doi:10.1186/1471-2334-10-233

Cite this article as: Tamayo et al: Spread of a highly mucoid Streptococcus pyogenes emm3/ST15 clone. BMC Infectious Diseases 2010 $10: 233$.

\section{Submit your next manuscript to BioMed Central} and take full advantage of:

- Convenient online submission

- Thorough peer review

- No space constraints or color figure charges

- Immediate publication on acceptance

- Inclusion in PubMed, CAS, Scopus and Google Scholar

- Research which is freely available for redistribution

Submit your manuscript at www.biomedcentral.com/submit 